\title{
PETRA OVERATH
}

\section{TRANSFER ALS VERENGUNG?}

\author{
Zur internationalen Diskussion über den Geburtenrückgang \\ in Frankreich in Texten von Fernand Boverat, \\ Roderich von Ungern-Sternberg sowie Joseph John Spengler \\ in den späten 30 er Jahren des 20 . Jahrhunderts ${ }^{1}$
}

\section{Distanzierte Blicke: Joseph John Spengler}

Im Jahre 1938 legte der Ökonom Joseph John Spengler von der Duke University, North Carolina (USA), ein Buch mit dem Titel »France faces depopulation « vor ${ }^{2}$. Es handelte sich dabei um die Ergebnisse eines umfassenden Forschungsprojekts Spenglers zur Bevölkerungsentwicklung in Frankreich und zugleich um den Auftakt für seine langjährige akademische Karriere, die sich bis in die neunziger Jahre des 20. Jahrhunderts erstrecken sollte ${ }^{3}$. Spengler stellte in seiner Studie zahlreiche Erklärungsversuche zusammen, die in Frankreich für das gefürchtete Phänomen des Geburtenrückgangs formuliert worden waren; ferner unterzog er die bislang ergriffenen staatlichen Maßnahmen zur Förderung der Geburtenrate einer eingehenden Prüfung. Um es gleich vorwegzunehmen: Spenglers Bewertung der Familien-, Sozial- und Geburtenpolitiken im Frankreich der vorangegangenen Jahrzehnte fiel vernichtend aus. Im letzten synthetisierenden Kapitel verwarf er nicht nur die Prämissen der staatlichen Maßnahmen zum Geburtenanreiz, sondern auch grundlegende Tendenzen der damals herrschenden gesellschaftlichen Ordnung. Er resümierte:

Letztlich ist die politische Philosophie der Pro-Populationisten in ihrer Substanz faschistisch. Sie begreift den gemeinen Mann als einen Organismus, welcher der >Wohlfahrt/ (welfare) des 'Staates oder der 'Nation< untergeordnet ist. [...] Dieser >Staat, so wie er jetzt organisiert ist, ist kein wahrhaftes Kollektiv, sondern ein von bestimmten Cliquen kontrolliertes

\footnotetext{
' Für Ideen und wertvolle Anregungen zu diesem Text danke ich Ursula Ferdinand, Werner Lausecker sowie den Teilnehmern des Workshops »Verräumlichung, Vergleich, Generationalität« in Leipzig, Juli 2004. Der Text benuht teilweise auf Vorarbeiten für das DFG-Projekt "Die Bevölkerung als wissenschaftliches Erkenntnis- und staatliches Verwaltungsobjekt. Zum Wissenstransfer in Bevölkerungsfragen (Deutschland und Frankreich, 1870-1945)«.

2 Joseph John SPENGLER, France faces depopulation, Durham 1938.

${ }^{3}$ Spengler lebte von 1902 bis 1991 . Weitere biographische Informationen unter www.econ. duke.edu/History/Spengler/Kelley.html.
} 
Instrument; die ,Wohlfahrt` des gegenwärtigen französischen 'Staates` ist vor allem die ,Wohlfahrt dieser Cliquen, aber nicht die des gemeinen Manns oder der Massen ${ }^{4}$.

Nach Ansicht Spenglers hatte der französische Staat grundlegend darin versagt, sinnvoll regulierend in die Bevölkerungsentwicklung einzugreifen. Mehr noch: Die Pro-Populationisten unterliefen aus seiner Sicht das parlamentarische System und drohten, Frankreich in die Reihe der faschistischen Staaten einzureihen. Spenglers Studie war in vielerlei Hinsicht ungewöhnlich. $\mathrm{Zu}$ gleich läßt sich seine wissenschaftliche Themenstellung, der Bevölkerungsrückgang in Frankreich, schon fast als konventionell bezeichnen. Immerhin hatten zahlreiche Wissenschafter, Politiker sowie engagierte Privatleute das Phänomen des Geburtenrückgangs kontinuierlich - wenn auch auf unterschiedliche Weise - bereits seit dem ausgehenden 19. Jahrhundert behandelt ${ }^{5}$. Entsprechend lag Ende der 1930er Jahre ein enorm umfangreiches Quellenmaterial sowie eine große Fülle von Sekundärliteratur zu diesem Themenkomplex vor, in denen der Geburtenrückgang aus verschiedenen disziplinären Perspektiven diskutiert wurde. Bei der Erörterung des Themas wurden, je nach Autor und dessen Position, höchst unterschiedliche Möglichkeiten staatlichen Handelns erörtert bzw. verschiedene in die Bevölkerungsentwicklung intervenierende Praktiken gefordert. Gleichwohl bestand ein breiter Konsens darüber, daß dem Staat die Verantwortung und Kompetenz für die Bevölkerungsregulierung oblag.

Im breiteren Spektrum der Studien über den Geburtenrückgang in Frankreich läßt sich eine Strömung ausmachen, deren Repräsentanten, für sich selbst Dominanz im wissenschaftlichen Feld beanspruchend, explizit innerhalb eines deutsch-französischen Referenzrahmens argumentierten, dessen Matrix das konfliktreiche, vor allem seit dem Krieg von 1870-1871 von Rivalitäten geprägte Verhältnis der beiden Staaten darstellte ${ }^{6}$. Spenglers Prämissen und Ergebnisse zeichneten sich dagegen durch eine explizite Distanzierung von diesem Rahmen aus. So vertrat Spengler die These, daß die Repopularisierungsdebatten in Frankreich in erster Linie der Verschleierung sozialer Ungleichheiten dienten und unabhängig von militärischen Erwägungen seien? ${ }^{\text {? In dem }}$

${ }^{4}$ SPENGLER, France faces, S. 299 (hier und im folgenden aus dem Englischen übersetzt von der Autorin).

${ }^{5}$ Grundlegend für die Zeit bis zum Ende des Ersten Weltkriegs: Christiane DIENEL, Kinderzahl und Staatsräson. Empfängnisverhütung und Bevölkerungspolitik in Deutschland und Frankreich bis 1918, Münster 1995; ferner: Petra OVERATH, Zwischen Krisendeutung und Kriegsszenarien. Bevölkerungspolitische Vorstellungen in Deutschland und Frankreich (1870-1918), in: DIES., Daniel SCHMIDT (Hg.), Volks-(An)Ordnung. Einschließen, ausschließen, einteilen, aufteilen!, Comparativ 3 (2003) S. 65-79.

${ }^{6}$ Claude DIGEON, La crise allemande de la pensée francaise (1870-1940), Paris 1959, besonders S. 74f.; OVERATH, Zwischen Krisendeutung, S. 66f.

${ }^{7}$ SPENGLER, France faces, S. 296; dazu auch: Remi LENOIR, Généalogie de la morale familiale, Paris 2003, S. 213. 
Segment von Geburtenrückgangstheorien, dessen Argumentationen auf einem deutsch-französischen Rivalitätsdenken beruhte, war dagegen die Position stark vertreten, daß ein Bevölkerungswachstum zu größerem wirtschaftlichen Wohlstand und damit $\mathrm{zu}$ mehr finanziellem Spielraum führe, um Kinder aufzuziehen. Ein Zuwachs an Kindern wurde wiederum mit einem größeren militärischen Potential der Nation korreliert. Führende Bevölkerungstheoretiker sowie Aktivisten der pronatalistischen Vereine nahmen das militärisch aggressive Deutschland, das die französische Nation zukünftig vermeintlich zu übermächtigen drohte, zum Ausgangspunkt ihrer Überlegungen. In diesem Sinne waren die Schriften bekannter Wissenschaftler und Aktivisten ausgerichtet, etwa die des Mediziners Jacques Bertillon, jene des Ökonomen Paul LeroyBeaulieu, des Soziologen Arsène Dumonts sowie die Pamphlete Fernand Boverats, der Vorsitzende des pronatalistischen Vereins L'Alliance nationale pour l'accroissement de la population française ${ }^{8}$. Spengler hingegen verwarf den Zusammenhang von quantitativer Bevölkerungsgröße und militärischer Verteidigungsstärke aus mehreren Gründen. Der Kern seiner Studie bestand in der differenzierten Widerlegung der Thesen, wonach ein Anwachsen der Bevölkerungszahl per se zu ökonomischem Wachstum und damit zur Stärkung der Wehrkraft der Nation führe. Er kritisierte das militärische Argument als "Kanonenfutter« zur Legitimation von bevölkerungspolitischen Maßnahmen, die letztlich lediglich das Besitzstanddenken der »haute bourgeoisie « verschleiern sollten?. Die deutsch-französischen Rivalitäten waren ihm zwar durchaus im Detail bekannt, aber er ließ sie nicht als zentrale Untersuchungsparameter für wissenschaftliche Arbeiten gelten. Im Gegenteil: Er dekonstruierte sie und legte deren ideologische Bedeutungen frei. Gleichwohl trat auch er für eine staatliche Regulation von Bevölkerungsentwicklungen ein. Diese hätte aber von demokratischen Prämissen auszugehen.

Spengler formulierte seine Position mit geographisch-politischer Distanziertheit. Er hatte an der Ohio State University bei dem Institutionalisten Albert B. Wolfe studiert, der sich schon früher für die Relationen zwischen Ökonomie und demographischen Entwicklungen interessiert hatte. Im Jahre 1934 ging Spengler an die wirtschaftswissenschaftliche Fakultät der Duke Universität, North Carolina, wo er rasch zu internationaler Bekanntheit gelangte ${ }^{10}$. Vermutlich erhielt Spengler die Anregungen zu seiner Studie von seinem akademischen Lehrer Wolfe. Zugleich stand er mit dem Demographen René Ku-

${ }^{8}$ Jacques BERTILLON, La colonisation de l'Europe par les Allemands, in: La réforme économique, revue des questions sociales, politiques, fiscales, scientifiques, industrielles, agricoles et commerçiales, 1 (1875) S. 154-169, S. 158f.; Paul LEROY-BEAULIEU, La question de la population, Paris 1913; Arsène DUMONT, Dépopulation et civilisation. Étude démographique, Paris 1890.

${ }^{9}$ SPENGLER, France faces, z.B. S. 129f., S. $256 f$.

${ }^{11}$ Als James B. Duke Professor der Ökonomie ging er 1972 in den Ruhestand, vgl. www. econ.duke.edu/History/Spengler/Kelley.html. 
czynski und dem Ökonom David V. Glass, die bereits wichtige bevölkerungswissenschaftliche Werke verfaßt hatten, in engem Kontakt ${ }^{\prime \prime}$. Spengler legte gemessen an der Strömung von Geburtenrückgangstheorien, die auf einem deutsch-französischen Rivalitätsdenken aufbauten - eine überaus differenzierte, in der Methodik ungewöhnliche und inhaltlich nuancenreiche Analyse vor. Auf der einen Seite nahm er die Frage der staatlichen Regulation der Bevölkerungsdichte sehr ernst, auf der anderen Seite entwickelte er grundsätzlich andere Sichtweisen und Kausalitäten, als sie innerhalb dieser Strömung des breiteren Spektrums von Geburtenrückgangstheorien kursierten. Obwohl sich Spenglers wissenschaftliche Kritik explizit auf dieses Segment der Geburtenrückgangstheorien bezog, rezipierten dessen Repräsentanten Spengler eher wenig. In diesem Teilbereich blieb die Studie längere Zeit fast unbeachtet. Die Gründe für diese lange sehr eingeschränkte Rezeption in diesem Feld können vielfältig sein. Erstens fand das Buch möglicherweise durch den Ausbruch des Zweiten Weltkriegs zunächst keine Verbreitung auf dem europäischen Kontinent. Zweitens mag auch die englische Sprache, in der das Buch abgefaßt war, für die Rezeption hinderlich gewesen sein. Drittens ist naheliegend, daß Spenglers Gedanken zumindest bis zum Ende des Zweiten Weltkriegs wenig kompatibel waren mit den Krisendiskursen sowie politischen Positionen dieser Strömung.

In den folgenden Abschnitten wird anhand von ausgewählten Texten dargelegt, daß der Wissenstransfer zwischen Akteuren in Deutschland und Frankreich zum Geburtenrückgang nicht unbedingt - wie man erwarten könnte - zu einer Verbreiterung der Palette von Argumentationen führte, sondern vielmehr erstarrte diskursive Strukturen sowie eine inhaltliche Engführung von Diskussionen tendenziell begünstigen konnte. In einem Teilbereich der Diskussion über den Geburtenrückgang stabilisierten sich Rezeptionskanäle und verfestigten sich Argumentationsmuster, die abweichende Positionen - wie jene Spenglers - inhaltlich kaum rezipierten: Einerseits funktionierte zwar bis zum Beginn des Zweiten Weltkriegs durchaus über nationale und politische Grenzen hinweg die wissenschaftliche Kommunikation zwischen an demographischen Fragestellungen interessierten Wissenschaftlern. Dies manifestierte sich nicht zuletzt während der Internationalen Kongresse. Andererseits basierte aber die imaginierte Internationalität auf nationalen Interessen, die staatenübergreifende Initiativen fragmentierten. Interessanterweise konnte dies, wie im folgenden gezeigt wird, zu Konvergenzen zwischen politischen Rivalen führen.

Anhand von Autoren, die verschiedene Geburtenrückgangstheorien präsentierten, wird in den folgenden Ausführungen eine mehrdimensionale Perspektive eröffnet. Gemeinsam ist den Akteuren ihre Themenstellung. Die Akteure

\footnotetext{
"SPENGLER, France faces, S. VIII; vgl. auch: David Victor GLASS, The Struggle for Population, Oxford 1936.
} 
werden im Folgenden allerdings nicht als Vertreter von nationalen Einheiten oder eines gesamten wissenschaftlichen Spektrums verstanden; vielmehr geht es darum, ihre jeweiligen Wissenschaftsverständnisse sowie ihre unterschiedlichen Projektionen nationaler bzw. internationaler Interessen herauszuarbeiten, die auch ihre Transferoffenheit beeinflußten. Bei den Autoren handelt es sich um Fernand Boverat, den langjährigen Vorsitzenden der Alliance nationale pour l'accroissement de la population française, den Ökonomen Roderich von Ungern-Sternberg sowie um den bereits eingeführten Joseph John Spengler. Sie standen mehr oder weniger intensiv in einem Austauschverhältnis und entwarfen verschiedene Perspektiven und ,Lösungsvorschläger hinsichtlich des `Problems` des Geburtenrückgangs in Frankreich. Im Gang der vorliegenden Untersuchung wird deutlich, wie sich in diesen mehrdimensionalen Räumen in den ausgehenden 1930er Jahren Zuspitzungen und Ausschlüsse in Transferprozessen entwickelten, die unterschiedliche Handlungsspielräume für den einzelnen Staat eröffneten oder eröffnen sollten.

\section{Geburtenrückgang als internationale Bedrohung?}

Ende Juli 1937 gaben sich Wissenschaftler und Politiker aus Frankreich als Gastgeber des Internationalen Bevölkerungskongresses. Die Kongreßteilnehmer tagten in Paris. Insgesamt nahmen knapp 300 Vertreter aus rund 30 Ländern an der Tagung teil, darunter zählte die französische Delegation als Gastgeber 86, die Delegation aus Deutschland 49 Mitglieder, gefolgt von Amerika (37), England (22), Italien (21), Holland (18), Belgien (14) und Österreich (6) sowie zahlreichen kleineren Delegationen ${ }^{12}$. Fernand Boverat, (vermutlich) einer der in der französischen Öffentlichkeit prominentesten Repräsentanten der französischen Vertretung, organisierte den Kongreß maßgeblich mit. Es handelte sich um die vierte Konferenz im Rahmen der International Union for the Scientific Investigation of Population Problems (IUSIPP), die während der Weltbevölkerungskonferenz in Genf als ein Dachverband von bevölkerungspolitisch orientierten Länderausschüssen geplant und im Jahre 1928 gegründet worden war $^{13}$.

${ }^{12}$ Horst GeYER, Der Internationale Kongreß für Bevölkerungswissenschaften in Paris, in: Der Erbarzt, 9 (1937) S. 124-125, S. 125.

${ }^{13}$ Bernhard vom BROCKE, Bevölkerungswissenschaft - Quo vadis? Möglichkeiten und Probleme einer Geschichte der Bevölkerungswissenschaft in Deutschland, Opladen 1998, S. 310f; diese Kongresse sind meines Wissens nach bislang noch nicht umfassend untersucht worden, Pionierarbeit leisteten u.a. Ursula FERDINAND, Bevölkerungswissenschaft und Rassismus. Die Internationalen Bevölkerungskongresse der International Union of the Scientific Investigation of Population Problems (IUSIPP) als paradigmatische Foren, in: Rainer MA- 
Fernand Boverat stand der bereits erwähnten privaten, einflußreichen Vereinigung Alliance nationale pour l'accroissement de la population viele Jahre als Generalsekretär vor. Die Mitglieder der Alliance, darunter zahlreiche Parlamentarier und Beamte, diskutierten seit der Vereinsgründung im Jahre 1896 die quantitative demographische Entwicklung Frankreichs und engagierten sich durch Propaganda sowie politische Lobbyarbeit für Maßnahmen zur Steigerung der Geburtenrate in Frankreich ${ }^{14}$. Durch ihre Personalstruktur existierten direkte Kontakte zwischen der Alliance und staatlichen Einrichtungen. Der Generalsekretär Fernand Boverat war zum Beispiel Vizepräsident des Hohen Rates der Natalität, der seit 1920 dem Ministerium für Hygiene, Fürsorge (assistance) und soziale Wohlfahrt untergeordnet und eine staatliche Schaltstelle zur Entwicklung von Maßnahmen gegen den Geburtenrückgang war. Die Alliance genoß seit 1913 den Status eines gemeinnützigen Vereins und verwaltete ein Jahresbudget von rund 500000 Francs. Mit diesen finanziellen Mitteln unterhielt der Verein ein Pariser Büro zur Koordination seiner breit gestreuten Aktivitäten, druckte Broschüren und schüttete Gelder direkt an kinderreiche bedürftige Familien aus. In der Zwischenkriegszeit zählte die Alliance zu den in der Öffentlichkeit sehr präsenten privaten Vereinen, die sich für Geburtenpolitik engagierten. Ende der 1930er Jahre, genauer im Juni 1939, stützte sie sich auf eine Mitgliederzahl von 25335 Personen's.

Boverat verfasste im Laufe seines langjährigen Engagements zahlreiche Bücher, Pamphlete sowie Broschüren über den Geburtenrückgang. Darin spielte der »Erbfeind" Deutschland, der sich aus Boverats Sicht einer deutlich günstigeren Bevölkerungsentwicklung erfreute als Frankreich, eine zentrale Rolle als Ausgangs- und Bezugspunkt sämtlicher Aktivitäten. Boverat trug mit der Alliance dazu bei, das Trauma vom verlorenen Krieg von 1870-1871 bis in die 1930er Jahre des 20. Jahrhunderts ebenso lebendig zu halten wie die Sorge, daß immer und überall die Gefahr einer »deutschen Invasion « bevorstehe. Vor allem seit der Machtergreifung der Nationalsozialisten und deren legislativen Aktivitäten interessierte sich Boverat explizit für die Geburtenpolitik Deutschlands. Mit eingängigen Formeln, wie »Geburtenrückgang - das ist der

2004, S. 61-98, hier vor allem 64f; Susanne HEIM, Berechnung und Beschwörung. Übervölkerung, Kritik einer Debatte, Berlin 1996, S. 25.

${ }^{14} \mathrm{Zu}$ Aktivitäten und Mitgliedern der Alliance vgl. Françoise THÉBAUD, Le mouvement nataliste dans la France de l'entre-deux-guerres: L'Alliance nationale pour l'accroissement de la population française, in: Revue d'histoire moderne et contemporaine 32 (1985) S. 276301; dazu auch Petra OVERATH, Zwischen Feindschaft und Familie. Eine Skizze zu Bevölkerungspolitiken in Frankreich vom Ende der III. Republik bis zur Vichy-Zeit, in: Rainer MACKENSEN (Hg.), Tagungsband zum Jahrestreffen der Deutschen Gesellschaft für Demographie, erscheint voraussichtlich 2006.

is ThÉBAUd, Le mouvement nataliste, S. 277-293; Éric JENNINGS, Discours corporatiste, propagande nataliste et contrôle social sous Vichy, in: Revue d'histoire moderne et contemporaine 4 (2002) S. 101-131, S. 108f;; OVERATH, Zwischen Feindschaft. 
Krieg«, machte er immer wieder auf die aus Deutschland drohende Gefahr einer übermächtigen Kinder- und Soldatenschar aufmerksam, von der das geburtenschwache Frankreich bald überrannt werden würde. Zur Rettung der französischen Nation forderte er vor allem staatliche finanzielle Anreize zur Steigerung der Geburtenrate. Aus seiner Sicht führte ein größerer Wohlstand zu einer gesteigerten Bereitschaft, Kinder aufzuziehen, und damit zu einer größeren Wehrkraft der Nation ${ }^{16}$.

Die Ressentiments gegen Deutschland spielten für Boverat freilich während des Kongresses in Paris kaum eine Rolle. Im Gegenteil: Hier ging es aus seiner Sicht um die Lösung eines Problems, des Geburtenrückgangs, das die westliche Welt allgemein betraf. Das leitende Paradigma des Bevölkerungskongresses bestand in der Frage, wie die westliche Welt dem Geburtenrückgang begegnen könne, um den Bedeutungsverlust der weißen Rasse zu vermeiden ${ }^{17}$. Entsprechend galt es, Erfahrungen auszutauschen sowie Gegenmittel zu diskutieren.

Die Diskussionen über den Geburtenrückgang hatten sowohl in Deutschland als auch in Frankreich eine längere Vorgeschichte, die hier nur kurz und vereinfacht und in einer Perspektive des Wissenstransfers umrissen werden kann: In Frankreich hatten sich zahlreiche Wissenschaftler und Politiker bereits seit dem 19. Jahrhundert mit dem Geburtenrückgang als "nationalem Problem« auseinandergesetzt. Sowohl in ökonomischen als auch in anderen wissenschaftlichen Feldern spielte die Untersuchung des Geburtenrückgangs eine wichtige Rolle. Im Deutschland des ausgehenden 19. Jahrhunderts hatten ganz ähnlich wie in Frankreich - verschiedene, vor allem ökonomische Strömungen existiert, die sich dem Zusammenhang von Wohlstand und Bevölkerungsentwicklung oder dem Problem der Übervölkerung widmeten ${ }^{18}$. Kurz vor dem Ersten Weltkrieg verstärkte sich die Tendenz einiger Wissenschaftler, wie zum Beispiel in Texten des Nationalökonoms, Mediziners und Sexualwissenschaftlers Julius Wolf ablesbar ${ }^{19}$, auch den Geburtenrückgang in Deutschland umfassend zu diskutieren. Schon bald darauf rückte die Lösung dieses Problems vor allem im Preußischen Staatsministerium auf die politische Agen$\mathrm{da}^{20}$. Diese Ausdifferenzierungen von Bevölkerungsfragen hatten vielfältige

\footnotetext{
${ }^{16}$ ThÉBAUd, Le mouvement nataliste, S. 279; Fernand Boverat, Comment nous vaincrons la dénatalité, Paris 1939, S. 6.

${ }^{17}$ FERDINAND, Bevölkerungswissenschaft und Rassismus, S. 86.

${ }^{18}$ Dazu ausführlicher HEIM, Berechnung und Beschwörung, v.a. S. $15 f$.

${ }^{19}$ Ursula FERDINAND, Geburtenrückgangstheorien in der Nationalökonomie Deutschlands zwischen 1900 und 1930. Fallbeispiel Julius Wolf (1862-1937), in: Rainer MACKENSEN (Hg.), Bevölkerungslehre und Bevölkerungspolitik vor 1933, Opladen 2002, S. 135-158, S. 146f., $151 f$.

${ }^{20}$ Paul WEINDLING, Die preußische Medizinalverwaltung und die "Rassenhygiene« 19051933, in: Achim THOM, Horst SPAAR (Hg.), Medizin im Faschismus. Symposium über das
} 
Ursachen und hingen unter anderem auch mit disziplinären Verschiebungen zusammen. Julius Wolf entwickelte zum Beispiel sexualwissenschaftliche Perspektiven auf Bevölkerungsfragen und machte dabei in seinem Werk $»$ Der Geburtenrückgang. Die Rationalisierung des Sexuallebens« im Jahre $1912^{21}$ auf das >Problem aufmerksam.

Eine nicht unwesentliche Rolle bei der zunehmend intensiv geführten Diskussion über den Geburtenrückgang in Deutschland vor dem Ersten Weltkrieg spielten auch die wachsenden zwischenstaatlichen Spannungen. Der französische Staatspräsident Poincaré verknüpfte beispielsweise seinerzeit den Geburtenrückgang explizit mit militärischen Erwägungen. Im Jahre 1912 setzte er eine parlamentarische Kommission zur Erforschung des Geburtenrückgangs ein, um vermutlich in der Öffentlichkeit bereits vorhandene Ängste zu kanalisieren und auf diese Weise die höchst umstrittene Einführung einer erweiterten, und zwar dreijährigen Wehrpflicht erfolgreich durchzusetzen. Einige Journalisten, Politiker und Wissenschaftler in Deutschland beobachteten diesen Vorgang mit großer Sorge und diskutierten verstärkt das Thema des Geburtenrückgangs nunmehr auch in Bezug auf die eigene Nation ${ }^{22}$. Dabei griffen sie eine Reihe von Erklärungsmustern aus Frankreich auf. Wissenschaftler aus Frankreich galten einigen Autoren als erfahrene Experten in der Frage des Geburtenrückgangs, weil sich diese bereits über vier Jahrzehnte damit auseinandergesetzt hatten. Mit dem Verweis auf den nationalen Rivalen machten Politiker in Deutschland entscheidende Schritte hin zu einer staatlichen Bevölkerungspolitik: Im Juni 1917 befürwortete das preußische Staatsministerium die Einrichtung eines ständigen Ausschusses für Bevölkerungspolitik, der im Abgeordneten-Haus angesiedelt wurde. Dessen Aufgabe bestand in der Ausarbeitung von Maßnahmen zum Geburtenanreiz. In die Weimarer Verfassung ging ferner die Verantwortung des Staats für die Bevölkerungspolitik $\mathrm{ein}^{23}$.

Bis zum 1937er Kongreß in Paris standen Übervölkerung, Geburtenrückgang sowie andere Bevölkerungsfragen in politischen und wissenschaftlichen Feldern in Deutschland auf spezifische Weise nebeneinander ${ }^{24}$. Auf der einen Seite nahm die Anzahl der Geburtenrückgangstheoretiker zu, auf der anderen Seite blieb das Übervölkerungsparadigma höchst aktuell. Beide Strömungen

Schicksal der Medizin in der Zeit des Faschismus in Deutschland 1933-1945, Berlin (Ost) 1985, S. 48-56, S. 49; OVERATH, Zwischen Krisendeutung, S. 78.

${ }^{21}$ Leipzig 1912.

${ }^{22}$ Vgl. dazu die Zeitungsartikel zusammengestellt in: Geheimes Staatsarchiv Preußischer Kulturbesitz, Rep. 76 VIII B Nr. 2011.

${ }^{23}$ Artikel 7 und 8 der Verfassung des Deutschen Reiches vom 31. Juli 1919. Nach den Beschlüssen der National-Versammlung in Weimar, Langensalza 1920, 1. Hauptteil; OVERATH, Zwischen Krisendeutung, S. 78.

24 Josef EHMER, Bevölkerungsgeschichte und Historische Demographie 1800-2000, München 2004, S $63 \mathrm{f}$. 
verbanden sich teilweise mit dem Denken über höher- und minderwertige Menschen. Vor allem während der Weltwirtschaftskrise nahm die Akzeptanz von eugenisch-selektierenden Maßnahmen deutlich $\mathrm{zu}^{25}$. Im Nationalsozialismus wurde dann die zum Teil in den Jahren zuvor diskutierten, zum Teil auch praktizierten menschenverachtenden Maßnahmen - wie zum Beispiel Sterilisationen - systematisch und in großem Umfang realisiert ${ }^{26}$.

Allerdings mehrten sich vor dem 1937er Kongreß unter den Wissenschaftlern im Ausland kritische Stimmen, die sich etwa gegen die Nürnberger Rassegesetze sowie die NS-Zwangssterilisationen richteten. Für die Bündelung antirassistischer nationaler Initiativen engagierten sich Persönlichkeiten wie Franz Boas, Ignaz Zollschan und die Mitglieder der Gruppe Races et Racisme aus Frankreich ${ }^{27}$. Entsprechend heftige Kritik ernteten auf dem Kongreß die Vertreter der deutschen Delegation, die durchweg aus systemtreuen Wissenschaftlern unter Ausschluß von Jüdinnen und Juden sowie Kritikern zusammengestellt worden war, mit der Präsentation von rassenhygienischen The$\operatorname{sen}^{28}$. Überhaupt provozierten vor allem die Vertreter der deutschen Delegation auch bei anderen Themen kontroverse Diskussionen ${ }^{29}$. Beispielsweise entwickelten sich in der Sektion zu den Ursachen des Geburtenrückgangs heftige Konfrontationen.

In einer dieser intensiven Debatten ergriff überraschend Fernand Boverat für die Repräsentanten der deutschen Delegation Partei, indem er ihnen zubilligte, daß gerade sie dem >Problem des Geburtenrückgangs umfangreiche staatliche Maßnahmen entgegenstellten. Der Präsident der populationistischen Vereinigung Alliance nationale, Boverat, der jahrelang die Notwendigkeit des Geburtenanreizes mit der Gefahr einer deutschen Invasion begründet hatte, verbündete sich in dieser Konfliktsituation spontan mit der Delegation des "Erbfeinds «. Dabei unterstützte Boverat allerdings weniger rassenhygienische Thesen. Vielmehr lief sein Plädoyer zur Unterstützung der Referenten aus Deutschland darauf hinaus, daß die bevölkerungspolitischen Schritte in Deutschland dazu beigetragen hätten, die »dépopulation« - ganz im Gegensatz

\footnotetext{
${ }^{25}$ WEINDLING, Die preußische Medizinalverwaltung, S. 49.

${ }^{26}$ Zur Ausarbeitung und Implementierung der Gesetze: Gisela BOCK, Zwangssterilisation im Nationalsozialismus. Studien zur Rassenpolitik und Frauenpolitik, Opladen 1986; HansWalther SCHMUHL, Rassenhygiene, Nationalsozialismus, Euthanasie. Von der Verhütung zur »Vermichtung lebensunwerten Lebens«, 1890-1945, Göttingen 1987.

${ }^{27}$ FERDINAND, Bevölkerungswissenschaft und Rassismus, S. 83; Congrès international de la population, Bd. VIII, Paris 1938. Ferner zur Kritik aus der französischen Delegation: Bundesarchiv Berlin-Lichterfelde, Bericht v. 1. Sep. 1937, Blatt $149 \mathrm{ff}$.

${ }^{28}$ GEYER, Der Internationale Kongreß, S. 125; Elisabeth PFEIL, Der Internationale Bevölkerungskongre $ß$ in Paris vom 28.7. bis 1.8., in: Archiv für Bevölkerungswissenschaft (Volkskunde) und Bevölkerungspolitik (1937) S. 288-301, S. 298f.; FERDINAND, Bevölkerungswissenschaft und Rassismus, S. 85.

${ }^{29}$ PFElL, Bevölkerungskongreß, S. 294.
} 
zum Vorgehen des französischen Staats - mit adäquaten Gegenmaßnahmen zu bekämpfen ${ }^{30}$. Mit anderen Worten: Seine Bewunderung entsprang der Anerkennung für die Effizienz und ,Ernsthaftigkeit der in Deutschland geführten Forschungen, den implementierten Maßnahmen und damit der aktiven Rolle des Staates, die er in der Regulation von Bevölkerungsentwicklungen einnahm.

Boverats Anerkennung galt dabei auch den Thesen des umstrittenen Roderich von Ungern-Sternberg, der in der Sektion zum Geburtenrückgang referierte. Ungern-Sternberg hatte mit seiner in englischer Sprache im Jahre 1931 veröffentlichten Arbeit »Die Gründe des Geburtenrückgangs im europäischen Kulturkreis« Bekanntheit erlangt und einen Preis der Eugenic Research Association in Long Island (New York) erhalten. Sein Spezialgebiet war somit die Erforschung des Geburtenrückgangs. In der preisgekrönten Schrift hatte Ungern-Sternberg seine Untersuchung vergleichend angelegt und der demographischen Entwicklung sämtlicher europäischer Länder jeweils ein Kapitel gewidmet. Sein Leben bestritt er als Schriftsteller und Privatgelehrter ${ }^{31}$.

Ungern-Stemberg galt in der Fachöffentlichkeit als Bevölkerungsexperte, den vor allem seine Kenntnisse über die (statistischen) Daten verschiedener europäischer Staaten auszeichneten. In seinen Studien hatte er auf der Ebene der internationalen Vergleiche von Bevölkerungslehren u.a. einen deutlichen Frankreich-Schwerpunkt: Zunächst legte er ein Buch zur Volkswirtschaft in Frankreich vor ${ }^{32}$. Später folgten weitere Monographien über den Nachbarn jenseits des Rheins. Vermutlich wurde Ungern-Sternberg aufgrund seiner Frankreichkenntnisse für die deutsche Delegation ausgewählt. Allerdings fand er dort keineswegs widerstandslos Aufnahme. Zwar läßt sich das exakte Aufnahmeverfahren in seinem Fall nicht lückenlos rekonstruieren; sicher ist aber, $\mathrm{da} B$ er erst im letzten Moment und als eher umstrittener Referent in die Delegation integriert wurde ${ }^{33}$. Vermutlich hing dies u.a. mit Schwierigkeiten von Seiten des Ministeriums zusammen, Ungern-Sternberg als ausreichend mili-

${ }^{30}$ PFEIL, Bevölkerungskongreß, S. 295; FERDINAND, Bevölkerungswissenschaft und Rassismus, S. 86.

${ }^{31}$ Vom BROCKE, Bevölkerungswissenschaft, S. 441.

${ }^{32}$ Roderich von UNGERN-STERNBERG, Die Volkswirtschaft Frankreichs, Berlin 1918.

${ }^{33}$ Eugen Fischer, Direktor des Kaiser Wilhelm-Institutes für Anthropologie, menschliche Erblehre und Eugenik, erstellte für das Reichs- u. Preußische Ministerium für Wissenschaft, Erziehung und Volksbildung bzw. für das Innenministerium eine Liste mit den von ihm präferierten Delegationsmitgliedern. Einzelne BewerberInnen, wie etwa Eugen Würzburger, engagierten sich selbständig oder auf Vermittlung von anderen WissenschaftlerInnen für die Teilnahme am Kongreß. In Absprache zwischen Fischer und den Ministerien erfolgte die Auswahl, wobei Würzburger als Jude und andere BewerberInnen als "politisch Unzuverlässige « abgelehnt wurden. Ungern-Sternbergs Name ist auf den Listen zwar durchgehend vermerkt, aber zumeist mit dem Zusatz versehen, daß ein Vortrag nicht erwünscht sei. Vgl. die Unterlagen in: Bundesarchiv Berlin-Lichterfelde, R 4901 Nr. 2760, Blatt $76 f$. 
tanten Vertreter von rassenhygienischen Forschungen, für die auf dem Kongreß geworben werden sollte, einzuordnen.

Untersucht man Ungern-Sternbergs Ausführungen über Frankreich genauer, dann fällt auf, daß sie in weiten Teilen nach dem gleichen Muster konzipiert waren, wie die Arbeiten des bereits seit den 1920er Jahren als Frankreichspezialist anerkannten Hans Harmsen, der ebenfalls an der Konferenz teilnahm ${ }^{34}$. Es entsteht der Eindruck, daß die Struktur ganzer Wissensbestände über Frankreich aus den Arbeiten von Harmsen übernommen wurde. Harmsen hatte in den 1920er Jahren zwei Studien über Bevölkerungsprobleme in Frankreich angefertigt. In seiner medizinischen Dissertation von 1924 hatte er eine deskriptive Auflistung von bevölkerungspolitisch orientierten Gesetzen in Frankreich angefertigt, die er als ein patriotisch-national ausgerichtetes bevölkerungspolitisches Programm interpretierte ${ }^{35}$. Mit der These von einer umfassenden, systematischen Bevölkerungspolitik, die der »Erbfeind « implementiere, hatte Harmsen die Konkurrenz mit dem militärischen Sieger genutzt, um in Deutschland »die Formung des Volkskörpers« durch Gesetze vorzubereiten. Zugleich hatte Harmsen die Intention, den politischen Feind herabzusetzen, indem er ihn als militärischen Sieger entwertete und als Opfer des Geburtenrückgangs darstellte. Die Demontage des Siegers in wissenschaftlichen Texten hatte nach der Niederlage Deutschlands im Ersten Weltkrieg und den Vereinbarungen des Versailler Vertrags kompensatorische und propagandistische Funktionen $^{36}$. Harmsen selbst hatte zu einer antidemokratisch-völkischen Strömung gezählt, die dem politischen System der Weimarer Republik ablehnend gegenüber gestanden war; seine revisionistischen Vorstellungen brachte er in wissenschaftlichen Arbeiten zum Ausdruck ${ }^{37}$. In seiner zweiten nationalökonomisch-philosophischen Dissertation über die Bevölkerungsprobleme Frankreichs von 1927 hatte Harmsen den Krisendiskurs fortgeführt.

Ungern-Sternberg griff eine Reihe von Untersuchungskategorien, die Harmsen entwickelt hatte, auf. In den Vordergrund stellte Ungern-Sternberg in der Arbeit von 1931 - genau wie zuvor Harmsen - regionale Differenzen des Geburtenrückgangs auf dem Territorium Frankreichs sowie die Frage der - wie es hieß - „Überfremdung«. Gleichwohl nahm er in sein Denken auch Kategorien auf, die Harmsen in seinen Arbeiten aus den 1920er Jahre weniger explizit behandelt hatte. So konstruierte Ungern-Sternberg seine Argumentationen

${ }^{34}$ Grundlegend über Hans Harmsen, Sabine SCHLEIERMACHER, Sozialethik im Spannungsfeld von Sozial- und Rassenhygiene: der Mediziner Hans Harmsen im CentralausschuB für die Innere Mission, Husum 1998.

${ }^{35}$ Hans HARMSEN, Die französische Sozialgesetzgebung im Dienste der Bekämpfung des Geburtenrückgangs, Berlin 1924, S. 32.

${ }^{30}$ Zum Versailler Vertrag: Detlev J. PEUKERT, Die Weimarer Republik. Krisenjahre der klassischen Modeme, Frankfurt a. M. 1987, S. 52-56.

${ }^{37}$ Die völkisch-antidemokratische Haltung Harmsens belegt ausführlich SCHLEIERMACHER, Sozialethik, S. 57, S. 59f. 
mit Raumvisionen, wobei er den "Lebensraum« als eigenständigen Faktor bei der Untersuchung von Bevölkerungsentwicklungen stark machte ${ }^{38}$.

Insgesamt liegt die Vermutung nahe, daß Ungern-Sternberg als FrankreichKenner im Schatten des längst etablierten Harmsen stand. Darüber hinaus war vermutlich Ungern-Sternbergs Profil für andere NS-loyale Wissenschaftler nicht immer eindeutig zuzuordnen. In seinem 1937 veröffentlichten Buch "Frankreich. Lebensraum und Wesen etwa $^{39}$, entwarf er zwar eine biologistisch orientierte Charakterologie, die auf der Grundlage einer Melange von phänomenologischen und rassischen Konstruktionen das - wie im Titel angekündigt - Wesen der Französinnen und Franzosen herausschälen sollte. Strekkenweise präsentierte sich das Buch aber auch als ein multidimensionaler Kulturführer Frankreichs, in dem Geschichte, Land und Leute fast wohlwollend beschrieben wurden. Gleichwohl enthielt die Analyse Ungern-Sternbergs eine Reihe von militärisch-politischen Einschätzungen, welche die ungewisse Zukunft der - aus seiner Sicht - durch den Geburtenrückgang geschwächten Nation manifestierten sollten. Diese Argumentationen machten Ungern-Sternberg vermutlich wiederum attraktiv für die deutsche Delegation.

Die militärischen Erwägungen hatten einerseits Frankreich entwertende Effekte, andererseits boten sie sich als Schnittstellen des Wissenstransfers an: Vor allem bei der Erkundung der militärischen, politischen und ökonomischen Zukunft des Landes griff Ungern-Sternberg Erklärungsmuster auf, in denen die Korrelation von Bevölkerungsentwicklung, Wohlstand und die Heeresstärke der französischen Nation zentral war. In diese Prognosen gingen spezifische Wissenselemente über den Nachbarn jenseits des Rheins ein. Dabei setzte auch Ungern-Sternberg voraus, ähnlich wie Boverat von der Alliance nationale, daß die Zunahme des wirtschaftlichen Wohlstands einer Nation den Kinderwunsch begünstige und damit das militärische Potential eines Landes bestärke. Parallel zu dieser Position existierten auch Strömungen, die davon ausgingen, daß gerade das Anwachsen ökonomischen Wohlstands die Einschränkung von Kinderwünschen zur Folge hatte. Aus dieser Perspektive führte das Bestreben, über die eigene soziale Position hinauszukommen, zu einem Verzicht auf Kinder. Ungern-Sternberg zählte - das mag fast paradox anmuten auch zu dieser Strömung: Seine These von »der streberischen Gesinnung « der Menschen, auf die zurückzukommen sein wird und die sich nicht eindeutig in vererbungsbiologische Paradigmen integrieren ließ, lief genau auf diese Argumentation hinaus ${ }^{40}$.

\footnotetext{
${ }^{38}$ Roderich von UNGERN-STERNBERG, Frankreich. Lebensraum und Wesen. Eine Einführung, Berlin 1937, S. 12.

${ }^{39}$ Ibid.

${ }^{40}$ Ursula FERDINAND, Systematisienung der Geburtenrückgangstheorien mum 1930«. Fallstudie für das DFG-Schwerpunktprogramm 1106, S. 5 f.
} 
Trotz vieler Unterschiede im Detail vertraten Ungern-Sternberg und Boverat auch vergleichbare Positionen. Zudem rezipierten sie sich gegenseitig; sie zählten zu einem Kreis von Personen, die Ende der 1930er Jahre ihre Studien auf die Beschreibung des Nachbarn jenseits des Rheins gründeten ${ }^{41}$. Das konfliktreiche Verhältnis der beiden Staaten hatte das Interesse an kontroversen Diskussionen ebenso wie Abgrenzungsinitiativen über lange Zeitspannen hinweg befördert ${ }^{42}$. In bezug auf den Wissenstransfer in Geburtenrückgangstheorien ist eine Tendenz deutlich: Aufgrund von ähnlich gelagerten Krisendiskursen, die sich seit 1900 herauskristallisiert hatten, ist für den Zeitraum von ca. 1900-1940 eine Transferoffenheit in bezug auf Geburtenrückgangstheorien zwischen Deutschland und Frankreich zu beobachten ${ }^{43}$. Vermutlich hatten die Krisendiskurse auf beiden Seiten - sehr allgemein gesprochen - eine integrative Wirkung: Sie boten affektive Elemente, die Männer und Frauen quer durch

${ }^{41}$ Inzwischen ist die Literatur zum Kultur- und Wissenschaftstransfer sehr umfangreich. Einen guten Überblick zum Transfer bieten verschiedene Artikel und Bücher, so etwa: Michel ESPAGNE, Michael WERNER, La construction d'une référence culturelle allemande en France - génèse et histoire (1750-1914), in: Annales E.S.C. juillet-aout (1987) S. 969-992; DIES., Deutsch-französischer Kulturtransfer im 18. und 19. Jahrhundert. $\mathrm{Zu}$ einem neuen interdisziplinären Forschungsprogramm des C.N.R.S., in: Francia. Forschungen zur westeuropäischen Geschichte, 13 (1985) S. 502-510; Matthias MIDDELL, Kulturtransfer und Historische Komparatistik - Thesen zu ihrem Verhältnis, in: Comparativ 1 (2000) S. 7-41, S. 18; jüngst: Hartmut KAELBLE, Jürgen SCHRIEWER (Hg.), Vergleich und Transfer. Komparatistik in den Sozial-, Geschichts- und Kulturwissenschaften, Frankfurt a. M., New York 2003; Michael Werner, Bénédicte ZIMMERmanN, De la comparaison à l'histoire croisée, Paris 2004; Zum Wissenschaftstransfer mit weiterführenden Literaturangaben vgl. Ralph JESSEN, Jakob VOGEL, Wissenschaft und Nation in der europäischen Geschichte, Frankfurt a. M., New York 2002.

${ }^{42}$ Dazu grundlegend: Michael JEISMANN, Das Vaterland der Feinde. Studien zum nationalen Feindbegriff und Selbstverständnis in Deutschland und Frankreich 1792-1918, Stuttgart 1992; Ulrich BIELEFELD, Nation und Gesellschaft. Selbstthematisierungen in Deutschland und Frankreich, Hamburg 2003. Die Literatur ist umfangreich; im folgenden werden nur einige wenige Studien exemplarisch herausgegriffen. Neben den bereits erwähnten Studien von Michael Werner, Michel Espagne und anderen zum Kulturtransfer sind für die vorliegende Fragestellung aufschlußreich: Laurent MUCCHIELLI, La guerre n'a pas eu lieu: les sociologues français et l'Allemagne (1870-1940), in: Espaces Temps 53-54 (1993) S. 6-18, vgl. S. 15f.; grundlegend sind auch: Hans Martin BOCK u.a. (Hg.), Entre Locarno et Vichy. Les relations culturelles franco-allemandes dans les années 1930, Band 1-3, Paris 1993; Jakob VOGEL, Nationen im Gleichschritt. Der Kult der »Nation in Waffen« in Deutschland und Frankreich, 1871-1914, Göttingen 1997.

${ }^{43}$ Für einzelne Disziplinen, Kultur- oder Politikbereiche liegen bereits umfassende Studien zu Transfer- und Blockadebewegungen zwischen Deutschland und Frankreich für das ausgehende 19. und die erste Hälfte des 20. Jahrhunderts vor, die Transferlogiken und -prozesse freilegen. Dennoch sind sie chronologisch gesehen ungleich verteilt: Deutlich mehr Arbeiten sind zum Kultur- und Wissenschaftstransfer vom 18. bis frühen 20. Jahrhundert als zu den folgenden $1920 \mathrm{er}$ bis $1950 \mathrm{er}$ Jahren erschienen. Dazu: Moritz FöllMER, Die Verteidigung der bürgerlichen Nation. Industrielle und hohe Beamte in Deutschland und Frankreich, 1900-1930, Göttingen 2002, S. 13f. 
alle sozialen Schichten in den Kampf für die Nation bzw. das Volk oder die Rasse einbinden sollten. Remi Lenoir entwickelte in diesem Zusammenhang für Frankreich die These, daß die Repopularisierungskampagnen der Krisenstimmung jener sozialen Kräfte ein Sprachrohr verliehen, die sich durch die organisierte Arbeiterbewegung bedroht sahen ${ }^{44}$. Mit diesen Kampagnen wurden auf Restriktion zielende Botschaften nicht nur an die erstarkende Sozialdemokratie, sondern auch an die Frauenbewegung gesendet, deren Aktivitäten auf den Wandel von traditionellen Ordnungsmustern hinausliefen. Diese Tendenzen existierten sowohl in Frankreich als auch in Deutschland, wenn auch in unterschiedlichen Ausprägungen. Vermutlich liegt in der Parallelität dieser oftmals diffusen Krisenstimmungen ein gewichtiger Grund dafür, daß der Wissenstransfer in der von Rivalitätsdenken bestimmten Strömung von Geburtenrückgangstheorien eine zentrale Rolle spielte ${ }^{45}$. Ferner spielte der Geburtenrückgang eine wichtige Rolle bei der Beurteilung der zwischenstaatlichen Beziehungen: Der Geburtenrückgang eröffnete eine imaginäre Projektionsfläche, vor deren Hintergrund das zwischenstaatliche Verhältnis bestimmt wurde. Die fast eindimensionale, komplexitätsreduzierende Verbindung von militärischer Stärke und Bevölkerungsgröße galt - bei Ungern-Sternberg und Boverat ebenso wie bei zahlreichen anderen Autoren - mehr oder weniger als selbstverständliche Prämisse und wurde kaum mehr hinterfragt. In diesen Fällen begünstigen Transferprozesse eine Verengung von Argumentationsspektren.

Im Gegensatz dazu verwarf Joseph John Spengler diesen Zusammenhang explizit. Als er seine wissenschaftliche Studie über die "dépopulation« in Frankreich konzipierte, lag ihm unter anderem das preisgekrönte Buch Ungern-Sternbergs aus dem Jahre 1931 als Grundlage vor. Auch die Arbeiten von Fernand Boverat waren ihm zugänglich, überhaupt kannte er die deutschfranzösische sowie englische Literatur der letzten 50 Jahre zum Geburtenrückgang offensichtlich sehr gut. Sein Anmerkungsapparat weist die Qualität einer sehr sorgfältig recherchierten Bibliographie des Schrifttums der Zeit zu Geburtenrückgangstheorien in Deutschland, Frankreich und darüber hinaus auf. Als Ausgangspunkt für seine umfangreiche Studie nannte Spengler eine tagesaktuell politische Motivation. »Insgesamt ist zu sagen: der aktuelle Alarm zur dépopulation ist, wenn auch keineswegs der erste in der historischen Erfahrung, der intensivste und sich am längsten hinziehende in der Geschichte Frankreichs ${ }^{46}$. Dies sah er vor allem in dem konfliktvollen Verhältnis der beiden Staaten begründet, das sich - so Spengler - erneut zuzuspitzen drohte. Spengler erkannte klar, daß Wissenschaftler sowie Politiker in Frankreich und

\footnotetext{
${ }^{44}$ LENOIR, Généalogie, S. $212 f$.

45 Dagegen zu Transferblockaden in den Naturwissenschaften: Brigitte SCHROEDERGUDEHUS, La science ignore-t-elle vraiement les frontières? Les relations franco-allemandes dans le domaine des sciences, in: BOCK, Locarno, S. 393-404.

${ }^{40}$ SPENGLER, France faces, S. 134.
} 
Deutschland die Definition ihres Verhältnisses zueinander in weiten Teilen über Erklärungen zum Geburtenrückgang aushandelten. Zugleich gab sich Spengler mit dieser Feststellung, auch wenn er militärische Drohgebärden vor allem das Expansionstreben des Hitler-Staats - an sich sehr ernst nahm, keineswegs zufrieden. Im Gegenteil: Die in Frankreich (und Deutschland) gängige Argumentation, daß eine kausale Beziehung zwischen Bevölkerungsziffer und militärischer Stärke existiere, führte ihn sogleich zu einem der fundamentalen Fehler, auf dem sämtliche Prämissen der populationistischen Bevölkerungslehren und -politiken in Frankreich seiner Sichtweise nach beruhten. Die französischen Populationisten - wie die Vertreter der Alliance nationale - argumentierten, so Spengler, in Kategorien der Kollektivität und vernachlässigten dabei gänzlich individuelle Faktoren, wie etwa jenen des individuellen Wohlstands ${ }^{47}$. Spengler hielt den Geburtenrückgang durchaus für eine zentrale politische Frage seiner Zeit; dabei kritisierte er aber die aus seiner Sicht in der französischen Bevölkerungspolitik angelegten faschistoiden Tendenzen sowie das Abrücken von einem demokratischen Wertekanon. Auf der politischen Ebene hatte die Behandlung des Geburtenrückgangs zudem eine internationale - weit über Frankreich hinausgehende - Dimension für Spengler: Ähnlich wie Boverat und Ungern-Sternberg setzte er sich für eine durchgreifende Staatsintervention zur Bevölkerungsregulierung ein. Grundlage seiner ökonomischen und demographischen Erwägungen bildete das Individuum, aber keinesfalls - wie bei den beiden anderen Autoren - ein Kollektiv wie Nation, Volk oder Rasse. Zwischen den drei Autoren bestanden demnach insofern Konvergenzen, als daß sie den Geburtenrückgang in Frankreich als ernste Bedrohung einordneten und dem Staat die Verantwortung für die Bevölkerungsregulierung übertrugen. Sie sahen den Geburtenrückgang als transnationales Phänomen und stimmten in der Problemdiagnose überein. Im einzelnen, das wird sich in den folgenden Abschnitten zeigen, wiesen ihre Argumentationen bei der vorgeschlagenen Problemlösung jedoch bemerkenswerte situationsbedingte Flexibiliäten sowie Divergenzen auf.

\section{Der Geburtenrückgang in Frankreich in dreifacher Perspektive}

Die Alliance nationale pour l'accroissement de la population verbreitete in ihren öffentlichen Kampagnen vornehmlich ein konservativ-reaktionäres Familienmodell. Insbesondere Fernand Boverat sprach sich in diesem Kontext für eine klassische Arbeitsteilung zwischen Mann und Frau aus. In diesen Kontexten begrüßte Boverat die nationalsozialistische Geschlechterpolitik, die

${ }^{47}$ SPENGLER, France faces, S. 9. 
Frauen aus der Erwerbsarbeit abziehen und ausschließlich in den Familienhaushalt einbinden sollte. Dazu zählte etwa die nationalsozialistische Initiative, den Arbeitsinspektoren aufzutragen, sämtlichen Frauen, die von einem arbeitlosen Mann ersetzt sowie vom eigenen Ehegatten ernährt werden könnten, den Ausstieg aus dem Arbeitsleben nahezulegen ${ }^{48}$.

Die Alliance überhöhte Familien zu Institutionen, die für die Zukunft des Landes verantwortlich seien. Ein Mitstreiter Boverats, Paul Haury, führte in diesem Sinne aus: "das was den Fortbestand der Nation garantiert, das ist die Familie, und nur diese allein «. Aus der besonderen Aufgabe, die der Familie zugeschrieben wurde, ergab sich, daß vor allem »der Familiensinn wieder hergestellt werden « müsse ${ }^{49}$. Kurze Zeit nach dem Internationalen Bevölkerungskongre $\beta$ in Paris ging Boverat gar soweit, den Familien die Verantwortung dafür aufzuerlegen, daß es nicht zum Krieg - mit Deutschland - komme. Unter dem polemischen Motto "Den Geburtenrückgang akzeptieren, das heißt den Krieg akzeptieren« führte Boverat im Namen der Alliance eine Kampagne gegen die wachsende Zahl freiwillig kinderloser Paare, die er für gefährlicher hielt für die Stabilität Frankreichs als die Armut. Er setzte sich für die allgemeine Verbreitung des »Familienglücks« und eine breite staatliche Unterstützung von kinderreichen Familien ein $^{50}$. Zum Erfolg verhalf der aggressiven Kampagne der Alliance, daß sie an einen Familiendiskurs anknüpfen konnte, der sich seit Gründung der III. Republik herausgebildet hatte. Die Hauptsorge des Gesetzgebers richtete sich seither auf jene Familien, die im Sinne des Gesetzes als arm galten. Dennoch setzte sich das staatliche Engagement für kinderreiche Familien eher langsam durch und wurde erst schrittweise, etwa mit einem geringen Steuerfreibetrag für Kinder sowie am 14. Juli 1913 mit einer "assistance obligatoire«, also einer flächendeckenden finanziellen Unterstützung für kinderreiche bedürftige Familien, realisiert ${ }^{51}$.

Die staatlichen bevölkerungspolitisch orientierten Maßnahmen in Frankreich waren divers und variierten zwischen unterstützenden und repressiven Maßnahmen. Ein im Jahre 1920 verabschiedetes Gesetz verbot zum Beispiel Abtreibungen sowie die Werbung für Verhütungsmittel und Kontrazeptiva

\footnotetext{
${ }^{48}$ Fernand BOVERAT, Extrait du Bulletin de l'Alliance nationale pour l'accroissement de la population française, décembre 1933, zitiert nach Francis RONSIN, La grève des ventres, Paris 1980, S. 206; OVERATH, Zwischen Feindschaft.

${ }^{49}$ Paul HaURY, Justice pour la famille ou la France est perdue, Paris 1938, S. $22 \mathrm{f}$.

${ }^{50}$ BOVERAT, Comment nous vaincrons, S. 6 u. 12.

51 Véronique ANTOMARCHI, La politique familiale en France sous la Troisième République (1870-1914), thèse microfiche, université Paris 1 1995, S. 390 u. 429; DIENEL, Kinderzahl, S. 28. In diesem Zusammenhang war auch die Bekämpfung der Säuglings- und Kindersterblichkeit zentral; vgl. dazu zum Beispiel die detailreiche Studie von Virginie DE LUCA, Les inspecteurs de l'assistance publique et la lutte contre la mortalité infanto-juvénile: Les causes et les moyens de leur engagement (1880-1914), in: Annales de démographie historique, 2 (1999) S. 137-170.
} 
selbst $^{52}$. Ungeachtet der Breite von Maßnahmen läßt sich gleichwohl bis zum Ende der 1930er Jahre eine zunehmende Tendenz zur Verstaatlichung der bevölkerungspolitischen Maßnahmen beobachten: Mit der Einrichtung eines Conseil supérieur de la natalité, dem regelmäßigen Abhalten von Congrès de la natalité sowie schließlich mit der Realisierung des Haut Comité de la population im Jahre 1939 entstanden eine Reihe von »offiziellen « Institutionen, in denen sich Staatsrepräsentanten mit den Ursachen sowie den Mitteln gegen den Geburtenrückgang beschäftigten. Fernand Boverat hatte in diesen Einrichtungen jeweils wichtige Funktionen inne und vermochte es, die Anliegen der Alliance auf staatlicher Ebene einzubringen. Vor allem Ende der 1930er Jahre, also in der Zeit des Internationalen Bevölkerungskongresses in Paris, genossen natalistische Vereine, wie die Alliance, breite Annerkennung ${ }^{53}$. Zugleich hielten sie das Feindbild des vermeintlich übermächtigen Deutschlands, aber auch die Faszination für den »Erbfeind« lebendig.

Die Faszination Boverats und der Alliance speziell für die NS-Bevölkerungspolitik entging auch Roderich von Ungern-Sternberg nicht. Bezug nehmend auf deren Kommentare diskutierte er den möglichen Einfluß politischer Systeme auf das Problem des Geburtenrückgangs in Frankreich und gelangte zu dem Ergebnis:

Ob parlamentarisch oder faschistisch-autoritativ regiert wird, ist nicht von wesentlicher Bedeutung für die Zukunft dieses Landes. Ja sogar ganz belanglos. Und darum können sich nur politische Eintagsfliegen über diese Dinge den Kopf zerbrechen. Das Schicksal des französischen Volkes vollzieht sich auf biologischem Gebiet. Entscheidend ist, ob die französischen Mütter das französische Volk davor bewahren werden, zusammenzuschrumpfen, ob in ihnen und damit in der ganzen Nation ein starker Selbsterhaltungstrieb wieder erwachen wird ${ }^{\text {s4 }}$.

In dieser Passage zeichnen sich Elemente ab, die sich kontinuierlich durch die Texte Ungern-Sternbergs in den ausgehenden 1930er Jahren zogen: Auf der einen Seite verwendete er biologistische - auch wie später gezeigt wird - rassistisch orientierte Erklärungsmuster, auf der anderen Seite betonte er die Bedeutung von Gesinnungen, Umwelteinflüssen und von individueller Verantwortung für den Geburtenrückgang. Er vertrat zu der Zeit eine spezifische Rassenkonzeption, die seine Bevölkerungskonstruktionen einerseits konstituierte und andererseits, und zwar durch das Hervorheben der wstreberischen Gesinnung « der Menschen hin zu sozialem Aufstieg, flexibilisierte ${ }^{55}$. Mit anderen Worten: In Texten Ungern-Sternbergs changierten Überlegungen zum Geburtenrückgang zwischen rassistischen und verhaltensorientierten Argu-

\footnotetext{
52 RoNSIN, La grève, S. $140 \mathrm{f}$.

${ }^{53}$ Paul-André RoSENTAL, L'intelligence démographique. Sciences et politiques des populations en France (1930-1960), Paris 2003, S. 17-34.

${ }^{54}$ Von UNGERN-STERNBERG, Frankreich, S. 131-132.

${ }^{55}$ FerdiNAND, Systematisierungen, S. $5 f$.
} 
mentationen. Damit waren zwar nicht sämtliche Bevölkerungsentwicklungen von rassistischen Merkmalen abhängig, aber die "sozial-biologische Einheit" eines Volkes - wie Ungern-Sternberg das nannte - wurde zum Orientierungspunkt erhoben, deren "künstliche Kleinhaltung « durch eine "rationalistische Einstellung zu natürlichen Vorgängen« eine ernstzunehmende Gefahr - und zwar des Bedeutungsverlusts oder gar der Existenzaufgabe - darstellte ${ }^{56}$. Ungern-Sternberg stellte einer behaupteten französischen materialistisch orientierten Gesinnung die bäuerlich »naturwüchsige« entgegen, von deren Aktivierung letztlich das Überleben der Nation abhängen sollte ${ }^{57}$. Offensichtlich kannte er die in Frankreich geführte Diskussion über den Geburtenrückgang sehr gut, griff dort präsente Erklärungsmuster, wie etwa den Wunsch nach sozialem Aufstieg oder die "streberische Gesinnung«, auf und verknüpfte sie mit rassistischen Konzeptionen.

Dabei sind allerdings Verschiebungen ablesbar. Im Unterschied zu seinen früheren Arbeiten hob Ungern-Stemberg vor allem seit dem Ende der 1930er Jahre generell die Bedeutung von Rassenanlagen, »die innerhalb eines Volkes als potentielle Kräfte von positiver und negativer Bedeutung zur Geltung kommen ${ }^{58}$, explizit hervor. Allerdings gründete er die jeweils wechselnde militärische Überlegenheit von Frankreich oder Deutschland nicht grundsätzlich auf divergierende Rassemerkmale; im Gegenteil, er betonte sogar Gemeinsamkeiten, und zwar dahingehend,

daß die Rassenzusammensetzung und mithin die körperlich-geistigen Kräfte bei Deutschen und Franzosen, wie ja die Anschauung lehrt, im hohen Grade die gleichen sind. [...] Die Blutsverwandtschaft ist, das verdient mit Nachdruck hervorgehoben zu werden, zwischen Deutschen und Franzosen sehr groß und einzelne Bestandteile des deutschen und des französischen Volks sind der Rassenabstammung nach untereinander viel näher verwandt als andere Bestandteile des eigenen Volkes ${ }^{59}$.

Den entscheidenden Unterschied zwischen den beiden Nationen machte er daran fest, da $\beta$ in Frankreich eine Atomisierung und Individualisierung des Menschen weit fortgeschritten sei, die das Land im politischen und ökonomischen Feld schwäche, wohingegen in Deutschland das Kollektiv an erster Stelle stehe. Von der Abkehr der noch aus der Zeit der französischen Revolution stammenden Prinzipien des Individualismus machte Ungern-Sternberg die Zukunft der wirtschaftlichen Entwicklung Frankreichs sowie dessen Existenz überhaupt abhängig. Nichtsdestotrotz erkannte er, fast neidvoll, auch die Erfolge dieser Prinzipien an:

\footnotetext{
${ }^{56}$ Von UNGERN-STERNBERG, Frankreich, S. 28.

${ }^{57}$ Ibid. S. $122 \mathrm{f}$.

${ }_{58}^{58}$ Ibid. S. 7.

${ }^{59}$ Tbid. S. 9 f.
} 
Es entsteht nun die Frage, wie bei der Atomisierung des Menschen in der französischen Gesellschaft, die sich als Folge der Ausbildung des Individualismus ergeben hat, Frankreich die Kräfte entwickeln konnte, um die französische Nation als Gemeinschaft zur Geltung zu bringen und einen nationalen Heroismus zu entfalten.

Als Antwort auf diese Frage verwies er auf den »citoyen«, dessen

Wesen nur auf einem ganz urwüchsigen völkischen Gemeinschaftsgefühl beruhen [kann], das seinerseits [auf] Instinkte sowie die Liebe zur heimatlichen Scholle (terre natale) und auf das tiefwurzelnde Solidaritätsbewußtsein, das in der gemeinsamen Sprache und in der Schicksals- und Kulturgemeinschaft verankert ist, zurückzuführen ist.

Daher stamme letztlich die Opferbereitschaft und der Heroismus, den Frankreich gegen Feinde an den Tag gelegt habe ${ }^{\infty}$. Mehr noch: Ungern-Sternberg verknüpfte die historisch gewachsene Einstellung "der Französinnen und Franzosen « mit der Vorstellung, daß sich Eigenschaften von Lebewesen veränderten und vererbten, so daß

das Wesen des französischen Volkes nicht nur durch Rassenanlagen und den jeweils vorherrschenden Zeitgeist, sondern auch durch die Umwelt [...] bestimmt wird ${ }^{61}$.

Bei Vertretern der Alliance spielte der von Ungern-Sternberg der Französischen Revolution zugeschriebene Individualismus ebenfalls eine, wenn auch eher marginale Rolle in der Diskussion der 1930er Jahre über den Geburtenrückgang. Der weithin bekannt Aktivist der Alliance nationale, Paul Haury, betonte, ähnlich wie Boverat, nicht nur die "großen Erfolge« Adolf Hitlers in der Bevölkerungspolitik, der - wie Haury meinte - an Frankreich sogleich mit einer doppelt so hohen Geburtenrate vorbeigezogen sei. Vielmehr entkräftete er auch das Argument, der französische Individualismus sei Schuld an der Misere der "dépopulation", die eine "wahre Degradierung der Lebensenergie» mit sich bringe. Um die wünschenswerte Qualität der Bevölkerung zu garantieren, habe der Staat durch geeignete Maßnahmen deren Quantität zu fördern. Der zentrale Hebel zur Realisierung dieses Ziels sei die Familie ${ }^{62}$. Auch wenn sich in den Geburtenrückgangsdiskursen der späten 1930er Jahre in Frankreich kaum negativ-eugenische Argumentationen durchsetzen konnten, so existierte gleichwohl eine Vision von der gesunden, moralisch und finanziell förderungswürdigen Familie. Die Repopulationisten verknüpften dabei nicht selten quantitative mit qualitativen Argumenten: Mit einer ausreichend hohen Geburtenrate sei, so das Denkmuster, die Wahrscheinlichkeit höher, ausreichend whochwertige«, gesunde Kinder zu generieren.

\footnotetext{
(4) Von UNGERN-STERNBERG, Frankreich, S. 91-92.

${ }^{61}$ Ibid. S. 93.

${ }^{62}$ HAURY, Justice pour la famille, S. $70 f$.
} 
Die Frage der Dauerhaftigkeit der französischen Nation beschäftigte auch Ungern-Sternberg. Allerdings strich er weniger die Bedeutung der Familie für die Bevölkerungsentwicklung heraus. Auf subtile Weise argumentierte er vielmehr, daß die Willensanstrengung zugunsten des Kollektivs die einzige wirkungsvolle Lösung des Problems der diffusen Bevölkerungsfrage sei. Bezogen auf das Problem des Geburtenrückgangs implizierten die Bevölkerungskonstruktionen von Ungern-Sternberg, daß die Rettung Frankreichs aus diesem Dilemma des Geburtenrückgang möglich sei, obwohl

die französische Nation nicht nur biologisch schwach ist, sondern daß auch alle Abwehrmittel gegen den biologischen Verfall, die bisher ergriffen worden sind, keine Abhilfe gebracht haben, und daß diese vorwiegend materiellen Maßnahmen wohl keine Wende in der natürlichen Bevölkerungsbewegung, im Sinne einer größeren Geburtenfreudigkeit herbeiführen können, weil die Kinderarmut gar nicht eine Folge wirtschaftlicher Notlage ist. Mit einer physiologischen Entartung, das sei hier nochmals festgestellt, hat der Geburtenschwund nicht das mindeste zu tun: er ist lediglich eine Auswirkung der geistigen Situation ${ }^{63}$.

Mit einem Mentalitäts- oder Weltanschauungswandel hielt er die Wende in der Bevölkerungsentwicklung Frankreichs demnach für möglich. Er betonte, daß die biologische Stärke durchaus in einem engen Zusammenhang mit der Willenskraft stehe und keineswegs zwingend "natürlich" vorgegeben sei. UngernSternberg projizierte die angenommene Stärke der »deutschen Volksgemeinschaft" auf Frankreich. Im Kern lief seine Argumentation auf die Behauptung hinaus, daß Frankreich zu seiner Rettung die gleiche Willenskraft aufbringen müsse, wie sie zuvor von der "Volksgemeinschaft« in Deutschland unter Beweis gestellt worden sei.

In späteren Texten, wie jenem aus dem Jahre 1938 zu den Bevölkerungsverhältnissen in Frankreich ${ }^{64}$, knüpfte Ungern-Sternberg an diese Überlegungen an. Er spitzte seine Gedanken allerdings zu, indem er Frankreich nun unmittelbar vom Untergang bedroht sah und seine Ausfïhrungen als vermeintliche Rettung für Frankreich ausgab. Ungern-Sternberg hob die Bedeutung der Erblehre hervor, die französische Wissenschaftler nicht ausreichend ernst nähmen. Er vertrat nunmehr ein positiv-eugenisches Konzept, für das er in Frankreich auf Zustimmung hoffen konnte, wonach eine hohe Geburtenziffer dazu beitrage, die positiven Merkmale eines Volkes zu mehren und die negativen in den Hintergrund treten zu lassen ${ }^{65}$. Er ging wiederum - ähnlich wie ein bedeutender Teil der Autoren in Frankreich - von der Prämisse aus, daß die Steigerung der Geburtenrate zu einer Verbesserung der militärischen und wirtschaftlichen Situation führe. Dabei sah er in der Perspektive eines erstarkenden Frankreichs

\footnotetext{
${ }^{63}$ Von UNGERN-STERNBERG, Frankreich, S. 116-117.

(4) DERS., Die Bevölkerungsverhältnisse in Frankreich. Eine sozialbiologische Studie, Berlin 1938.

${ }^{65}$ Ibid. S. $45-46$.
} 
keine unmittelbare Bedrohung für Deutschland. Vielmehr legte er den Akzent auf die Stärkung der »westlichen Zivilisation«. Er begrüßte die in Frankreich ergriffenen Initiativen, die der Erblehre eine größere Bedeutung einräumten ${ }^{66}$. Dabei gelangte Ungern-Sternberg auch durchaus zu differenzierten Ergebnissen, wonach »Rasse« in Frankreich in der Regel gänzlich anders als in Deutschland gebraucht werde und vielmehr synonym mit Volk sei ${ }^{67}$. Gleichwohl implizierte die Hervorhebung der Bedeutung der Erblehre auch ein Überlegenheitsdenken: Ungern-Sternberg zeichnete ein Bild, wonach Deutschland in bevölkerungspolitischen Fragen Vorreiter sei und Frankreich hinterher hinke. Solange die französische Nation nicht auf effiziente Maßnahmen zur Bekämpfung des Geburtenrückgangs umschwenke - und das hieß: die Rassenfrage und Erblehre einbezog -, solange würden sie auch keine dauerhaften Erfolge verzeichnen können.

Bis zum Zweiten Weltkrieg behielt Ungern-Sternberg die Vision von einem zumindest wissenschaftlichen Schulterschluß zwischen Deutschland und Frankreich lebendig. In weiten Teilen verknüpfte er in seinen Bevölkerungskonstruktionen wesentliche Elemente aus den Diskussionen in Frankreich und in Deutschland. Die Verbindung von Mentalitäts- mit biologistisch-rassistischen Faktoren ermöglichte eine hohe Flexibilität in der Argumentation. Ungern-Sternberg ging über die in Frankreich vorherrschende Vorstellung hinaus, daß die Familie im Mittelpunkt bevölkerungspolitischer Maßnahmen stehen müsse. Er präsentierte ein Rassekonzept, wonach die Vitalität und die Qualität der Erbanlagen einer Nation durch Mentalität und Gesinnung beeinflußbar schienen. Gleichwohl ließ Ungern-Stemberg immer wieder durchblicken, daß die Orientierung der Bevölkerungspolitik an Rasse und Erbanlagen, wie in Deutschland der Fall, wesentlich erfolgversprechender war als die Orientierung an familialen Strukturen. Mit dem Ausbruch des Zweiten Weltkriegs spitzte Ungern-Sternberg seine Thesen noch weiter zu. Nunmehr deklarierte er Frankreich offen als eine im Verfall begriffene Nation. In seinen zunehmend rassistisch orientierten Bevölkerungskonstrukten transportierte er militärische Propaganda, die sich gegen den Rivalen Frankreich richtete ${ }^{68}$. Mit der verstärkten Verwendung von rassistischen Überlegungen schränkte sich die Argumentationsbreite in seinen Geburtenrückgangstheorien zunehmend ein.

Obwohl Ungern-Sternberg die differenzierte Arbeit von Joseph John Spengler kannte, zitierte und ebenfalls mentalitätsgeschichtliche Faktoren in Rechnung stellte, setzte er sich nur indirekt mit dem kritischen Potential der dort formulierten Thesen auseinander, die unter anderem auf die faschistoiden

\footnotetext{
${ }^{60}$ Von UNGERN-STERNBERG, Bevölkerungsverhältnisse, S. 34-35.

${ }^{67}$ Ibid. S. 35 .

${ }^{18}$ Roderich von Ungern-STERnBerg, Der volksbiologische Verfall in Frankreich, Berlin 1940.
} 
Tendenzen der Bevölkerungspolitiken in Frankreich verwiesen ${ }^{69}$. UngernSternberg prüfte durchaus, wie oben ausgeführt, den Einfluß politischer Systeme auf Bevölkerungsregulierungen. Diesen Faktor erklärte er aber für unwirksam und hob stattdessen die Bedeutung von biologischen Entwicklungen hervor, die wiederum durch die Gesinnung der Menschen, insbesondere jene der Frauen, lenkbar schienen. Er opponierte demnach keineswegs gegen biologistische NS-Rassen-Ideologien; zugleich baute er in seine Theorien aber Elemente ein, wie etwa den Zusammenhang von Mentalität und Geburtenrate, die für Bevölkerungstheoretiker aus Frankreich wie Boverat annehmbar schienen. Mit dem Beginn des Zweiten Weltkriegs radikalisierte sich dann die antifranzösische Stoßrichtung Ungern-Sternbergs.

Die Besonderheit der Studie Spenglers bestand darin, daß sie zentrale Prämissen der Bevölkerungslehren seiner Kollegen in Frankreich und Deutschland dekonstruierte und damit die Ausgangspunkte vieler Arbeiten zum Geburtenrückgang - etwa jene von Boverat und Ungern-Sternberg - grundlegend in Frage stellte. Zum einen führte er den Nachweis, daß ein Anwachsen der Bevölkerungsziffer keineswegs zwingend zu einem breiten Anwachsen des Wohlstands und damit zur gesteigerten Bereitschaft führe, Kinder aufzuziehen, die wiederum für die militärische Wehrkraft zentral seien. Zum anderen widerlegte er die in Frankreich vorherrschende Ansicht, daß die Orientierung auf die Familie der geeignete Ansatzpunkt sei, um die Bevölkerungsentwicklung sinnvoll zu regulieren. Spengler machte somit ein Angebot, um die seit Jahrzehnten eingefahrenen Argumentationsstrukturen, die sich zwischen einigen Akteuren im deutsch-französischen Wissenstransfer herausgebildet hatten, aufzubrechen. Aber die Rezeption dieser Argumente hätte eine wissenschaftlich-politische Umorientierung sowie das Hinaustreten über das Feindschaftsund Rivalitätsdenken erfordert, wofür jedoch offensichtlich weder Boverat noch Ungern-Sternberg offen waren. Aus der Sicht Boverats und UngernSternbergs waren die wissenschaftlichen Thesen Spenglers durch die Kritik an autoritären Staatsystemen diskreditiert. Zugleich bildete das nationale Konkurrenzdenken für Boverat und Ungern-Sternberg einen geeigneten Rahmen, um die Bewunderung für das jeweils andere politische System formulieren zu können.

Die gängigen, damals weithin anerkannten wissenschaftlichen Thesen, die von einer ökonomischen und militärischen Schwächung durch den Geburtenrückgang in Frankreich ausgingen, widerlegte Spengler in fünf höchst differenzierten Untersuchungsschritten. Erstens stellte er kompilatorisch sämtliche wissenschaftlichen Erklärungen des Geburtenrückgangs in Frankreich zusammen. Im Wesentlichen verwies er auf die verschiedenen Varianten von Theo-

\footnotetext{
${ }^{69}$ Roderich von UNGERN-STERNBERG, Frankreich. Kleine Frankreichkunde, hg. vom deutschen Auslandswissenschaftlichen Institut, Berlin 1943, S. 19.
} 
rien, die den Willen zum sozialen Aufstieg als Grund für den Verzicht auf Kinder stark machten, verwies ferner auf ökonomische Erklärungen, wonach zahlreiche Familien durch Kinder in Finanznöte gerieten sowie auf Polemiken gegen den erstarkenden Feminismus ${ }^{70}$. In einem zweiten umfangreichen $\mathrm{Ab}-$ schnitt diskutierte er das Datenmaterial sowie die politischen Prämissen der wirtschaftlichen Theorien. Seine erste Kritik galt der empirischen Basis, auf deren Grundlage bislang Aussagen über das Verhältnis von Fruchtbarkeit und Wohlstand getroffen worden waren. Die für Frankreich zur Verfugung stehende Datenmenge bewertete Spengler als unzureichend für gesicherte wissenschaftliche Aussagen. Dementsprechend beschränkte er sich darauf, aus den empirischen Daten lediglich Tendenzen abzuleiten, die er als noch nicht ausreichend gesicherte wissenschaftliche Aussagen - wie er explizit betonte - zur Diskussion stellte. Im Blick auf die differentielle Fruchtbarkeit um 1938, also die Geburtenhäufigkeit in verschiedenen sozialen Schichten, beschrieb er beispielsweise die Tendenz, daß die Fruchtbarkeit der "employé(e)s« und der Arbeiterschaft deutlich stärker sinke als die der Klasse der Eigentümer. Entgegen der gängigen Behauptungen sei der soziale Druck auf die Arbeiterklasse besonders stark ${ }^{71}$. Aufgrund dieser Tendenzen sowie der gesicherten Ergebnisse aus anderen Staaten, betonte Spengler, daß ein Anwachsen der Bevölkerung eben nicht zu einem größeren Wohlstand der Individuen führe. Ebenso wenig führe ein Sinken der Bevölkerungsziffer zwingend zu einer wirtschaftlichen Depression. Vielmehr spielten nicht-demographische Faktoren, wie zum Beispiel das Bildungssystem, die Arrangements zwischen Arbeitgebern und -nehmern sowie der Umgang mit den anderen Produktionsfaktoren, die entscheidende Rolle sowohl hinsichtlich einer sinnvollen Verteilung von Wohlstand wie auch in bezug auf demographische Entwicklungen ${ }^{72}$.

An dieser Stelle führte Spengler drittens den Staat als notwendig regulierende Instanz der Bevölkerungsentwicklung ein. Obwohl er Staatsinterventionen in diesem Politikfeld ähnlich wie Ungern-Sternberg und Boverat für unvermeidlich hielt, stellte er zentrale Widersprüche heraus, die sich in Frankreich abzeichneten. Im Kern argumentierte er, daß die »Ideologie der großen Bevölkerung« der Wirtschaftskraft der whaute bourgeoisie« sowie der Nobilität zugute käme. Sämtliche staatlichen Maßnahmen zur Bekämpfung des Geburtenrückgangs beruhten auf der Annahme, daß finanzielle Unterstützungen die Familien motivierten, mehr Kinder in die Welt zu setzen. Das Anwachsen der Bevölkerung führe letztlich aber zu einer Schwächung der Arbeiterschaft durch Rückgang der Löhne, was die Stellung der oberen Klassen stärke. Die bislang in Frankreich eingeführten staatlichen Maßnahmen konzentrierten sich viertens auf die Familie. Da sich die Rolle der Familie jedoch in den letzten

\footnotetext{
${ }^{70}$ SPENGLER, France faces, S. $168 \mathrm{f}$.

${ }^{7}$ Ibid. S. 7f., S. 95-102, S. 127 f.

${ }^{72}$ Ibid. S. $257 \mathrm{f}$.
} 
Jahrzehnten durch einen tiefgreifenden Mentalitätswandel von einer agrarischen Wirtschaftseinheit hin zu einem Beziehungssystem zum Ausleben von intimen Affekten gewandelt habe, könne die Familie höchstens dann erfolgreich als Institution zur Hebung der Geburtenrate funktionieren, wenn ausreichende finanzielle Mittel seitens des Staates zur Verfügung stünden, die Familie zu subventionieren. Im Anschluß daran errechnete er die unter diesen Voraussetzungen notwendige Summe, die für sämtliche Familien mit Kindern ein auskömmliches Leben ermöglichen würde. Da diese Summe beträchtlich war und etwa ein Drittel des gesamten jährlich erwirtschafteten Einkommensvolumens betrug, kam er zu dem Schluß, daß vor allem der »surplus«, also das über das zum Leben Notwendige im beschriebenen Sinn Hinausgehende, der besonders hohen Einkommen weitgehend abgeschöpft werden müsse. Gleichwohl hielt er das für unmöglich und damit die Familie auch nicht für den geeigneten Hebel zur Bekämpfung des Geburtenrückgangs: "Da ein Abschöpfen der surplus Werte der höheren Einkommensklassen zur Zerstörung von deren ökonomischer und politischer Macht führen wird, werden sie das zu vermeiden suchen, was wiederum den Klassenkampf intensivieren wird ${ }^{73}$. Für den sehr unwahrscheinlichen Fall, daß die Gelder aber doch auf diese Weise verteilt würden, prognostizierte Spengler eine höchst gefährliche Entwicklungstendenz, die George Orwells »1984« nahe kam: Der Einsatz von Geldern der oberen Einkommensklassen führe zu einem Rechtfertigungsdruck über die verwendeten Gelder und gleichzeitig zu einem Sparzwang. Sobald sich herausstelle, daß die Kindererziehung in Familien teurer als in staatlichen Institutionen unter Einsatz von modernen technischen Mitteln sei, verlöre die Familie die Funktion der Kindererziehung. Dem Staat falle zugleich die schwierige Aufgabe zu, immer größere Geldsummen abschöpfen zu müssen. Dies führe erneut und zu verstärktem Sparzwang, dem der Staat nur durch Auswahlsysteme begegnen könne. In diesen Auswahlsystemen würden nur einige wenige Frauen das Recht erhalten, Kinder zu bekommen, wobei letztere dann in staatlichen Institutionen erzogen würden. Schließlich würde das entscheidende Kriterium zur Auswahl der Mütter der genetische Code sein und entsprechend nur als genetisch hochwertig erachtete Frauen ausgewählt wer$\operatorname{den}^{74}$.

Spengler dekonstruierte demnach nicht nur die wissenschaftlichen Prämissen der Geburtenrückgangstheorien zu Frankreich, vielmehr legte er auch deren, wie er es nannte, faschistoides Potential frei. Aus seiner Perspektive bargen die natalistischen und familialistischen Bevölkerungskonstruktionen die Tendenz, sich mit einer autoritär-faschistischen Gesellschaftsordnung zu verbinden, indem sie das Individuum einer übergeordneten Größe, wie etwa der

\footnotetext{
${ }^{73}$ SPENGLER, France faces, S. 288f., $298 \mathrm{f}$.

${ }^{74}$ Ibid. S. $291 \mathrm{f}$.
} 
Nation oder Rasse, unterwarfen. Insofern war Spenglers Studie nicht nur eine komplexe Analyse des bevölkerungspolitisch engagierten wissenschaftlichen Feldes in Frankreich; sie beinhaltete auch politische Weitsicht sowie eine Warnung, die sich auf die Grenzen der leistbaren und wünschenswerten Kontrolle von Bevölkerungsentwicklungen durch den Staat bezog. Seine Projektionsfläche zur Diskussion des Geburtenrückgangs in Frankreich war ein demokratischer Wertehorizont, den er in Frankreich gefährdet sah. Um einen Weg zur Umkehr aufzuzeigen, entwickelte er die Vision eines sozialen Systems, in dem der einzelne Mensch nicht länger dem Staat oder der Nation als »dienender Organismus « untergeordnet war $^{75}$. Um dieses System tatsächlich zu realisieren, forderte er eine Änderung der kulturellen Muster in Frankreich ein, die ein neues System von Leistung und Lohn etablierten und zu einer grundlegenden sozialen Angleichung zwischen den Menschen führen müßte. Denn nur in diesem Falle, so Spengler, erhöhe sich die Chance, daß die Bevölkerungsentwicklung in Frankreich erfolgreich staatlich regulierbar sei. Allerdings bliebe es dabei, daß die staatlichen Stimulanzen letztlich bei jedem Menschen einen anderen Effekt hätten ${ }^{76}$.

\section{Schlußbetrachtung}

Die von Boverat, Ungern-Sternberg und Spengler zur Diskussion gestellten Formulierungen von Geburtenrückgangstheorien sowie die vorgeschlagenen Lösungswege entstanden in Kontexten des Wissenstransfers und teilweise auch in Kontexten von zwischenstaatlichem Rivalitätsdenken. Die drei Autoren forderten eine, allerdings unterschiedlich weit gehende, staatliche Regulierung der Bevölkerungsentwicklung.

Argumentationen, wie jene von einer angeblichen Korrelation von Bevölkerungsgröße und militärischer Stärke, waren über viele Jahre hinweg höchst stabil und behinderten den Bezug auf abweichende Positionen, wie sie Spengler vertrat. Vor allem waren Spenglers wissenschaftliche Thesen aus der Sicht von Boverat und Ungern-Sternberg durch dessen Kritik an autoritären Staatssystemen diskreditiert. Zwischen Ungern-Stemberg und Boverat kristallisierten sich, trotz der tradierten politischen Feindschaft bzw. Rivalität zwischen ihren Herkunftsstaaten sowie zentraler Unterschiede in der Argumentation, auch Konvergenzen im Hinblick auf ihre wissenschaftlichen Positionen heraus, die sich flexibel-situativ wandelten. In Kontexten von behaupteten oder tatsächlichen militärischen Bedrohungen trugen sie - trotz der in ihnen enthal-

\footnotetext{
${ }^{75}$ SPENGLER, France faces, S. 297.

${ }^{76}$ Ibid. S. $282 \mathrm{f}$.
} 
tenen flexiblen Elemente - zur Verengung der Argumentationsbreite bei der wissenschaftlichen Thematisierung von demographischen Perspektiven bei. Jenseits nationaler Rivalitäten bestanden nicht nur wissenschaftliche, sondern auch politische Affinitäten zwischen Boverat und Ungern-Sternberg: Ohne sich gänzlich zur NS-Bevölkerungspolitik zu bekennen, sich von einzelnen staatlichen Praktiken, wie etwa Zwangssterilisationen, explizit abgrenzend, fixierte sich Boverat bei der Formulierung von Bevölkerungsfragen auf Deutschland und unterstützte den nationalen Rivalen sogar auf dem Internationalen Bevölkerungskongreß von 1937 argumentativ. Umgekehrt äußerte sich auch Ungern-Sternberg, jenseits zahlreicher politischer Entwertungsversuche, immer wieder anerkennend in Hinsicht auf die republikanischen Staatstraditionen des französischen Nachbarn. Im Rahmen von nationalem Rivalitätsdenken konnten sich, wie gesehen, durchaus Konvergenzen und spezifische Transferkanäle herausbilden, die über politische Systemgrenzen hinausgingen.

Boverat, Spengler und Ungern-Sternberg forcierten interventionistische staatliche Politiken zur Hebung der Geburtenrate. Während Boverat die Familie als Objekt staatlichen Handelns favorisierte, machte Ungern-Sternberg kollektive Mentalitäten sowie Rasseanlagen als Ausgangspunkte für Bevölkerungspolitiken stark. Auch Spengler befürwortete, wie gesagt, eine staatliche Regulierung der Bevölkerungsentwicklung und bezog Mentalitäten als zentralen Faktor in seine Untersuchungen des Geburtenrückgangs mit ein. Allerdings vertrat er im Gegensatz zu Boverat und Ungern-Sternberg eine dezidiert kritische Position zu - von ihm als faschistoid betrachteten - Strategien staatlichen Handelns, die Individuen kollektiven Interessen unterzuordnen versuchten. Spengler markierte demnach, im Gegensatz zu Boverat und UngernSternberg, deutlich die Grenzen staatlicher Einflußmöglichkeiten in staatlichen Geburtenpolitiken.

Längerfristig spielte Boverats' Alliance nationale, etwa bei der Verbreitung von natalistischer und familialistischer Propaganda, eine wichtige Rolle in der Geburten- und Familienpolitik der Vichy-Zeit. Letzteres geschah allerdings ohne Mitwirkung Boverats, der im Juni 1940 seinen Platz als Generalsekretär der Alliance räumte. Offensichtlich hatte er sich von seinen Mitstreitern aufgrund seiner autoritären Haltung entfremdet ${ }^{77}$.

${ }^{77}$ RoSENTAL, L'intelligence, S. 61. 


\section{Résumé français}

Dans le vaste éventail d'études qui ont abordé la question du déclin de la natalité en France, il existait, vers la fin des années 1930, un courant dont les représentants argumentaient à l'intérieur d'un cadre de référence franco-allemand dont la matrice était constituée par la relation conflictuelle des deux États, en situation de rivalité surtout depuis les guerres de $1870 / 71$ et de 1914/18. À partir de positions scientifiques et politiques différentes de part et d'autre, on y discutait, sur le mode de la controverse, de la possibilité et des limites d'une intervention étatique pro-nataliste.

Dans le présent article, l'auteur constate, à partir d'un choix de textes, qu'en ce qui concerne le déclin de la natalité, le transfert de savoir entre acteurs allemands et français n'a pas nécessairement débouché sur un élargissement de la palette des argumentations - comme on pouvait l'espérer - mais a plutôt eu tendance à paralyser la structure de l'argumentation et à en appauvrir le contenu de la discussion. Dans un secteur du débat, les canaux de réception se figèrent et des modèles d'argumentation ne prenant pas en compte d'éventuelles positions divergentes se renforcèrent. Par delà les rivalités nationales se constituèrent néanmoins, à cette occasion, des convergences non seulement scientifiques, mais aussi politiques. En analysant les textes des économistes Joseph John Spengler et Roderich von Ungern-Stemberg, ainsi que de Fernand Boverat, Président de l'association de droit privé »Alliance nationale pour l'accroissement de la population française «, l'auteur met en relief les coïncidences thématiques, les mécanismes d'exclusion, mais aussi les imputations en ce qui concerne l'intervention de l'État pour une ssolution` du sproblème` du recul des naissances en France. Il apparaît alors clairement que tous les trois considéraient le déclin de la natalité comme un phénomène transnational et prenaient position pour une régulation étatique de l'évolution démographique qui devait, néanmoins, prendre des dimensions et des formes extrêmement diverses. 
